\title{
DIFFERENTIAL OPERATORS ON MODULAR FORMS ASSOCIATED TO THETA SERIES
}

\author{
Min Ho LeE
}

\begin{abstract}
We construct an operator on modular forms by modifying the derivative operator using certain theta series. To show that the image of a modular form under this operator is a modular form we use Jacobi-like forms determined by theta series as well as quasimodular forms obtained from such Jacobi-like forms.
\end{abstract}

Keywords: modular forms, theta series, Jacobi-like forms, quasimodular forms

\section{Introduction}

As is well-known, modular forms for a discrete subgroup of $S L(2, \mathbb{R})$ plays an important role in number theory. Although the derivative of a modular form is not modular in general, there is a modified derivative operator carrying a modular form to a modular form constructed as follows. Let $M_{k}\left(\Gamma_{1}\right)$ be the space of modular forms of weight $k$ for the modular group $\Gamma_{1}=S L(2, \mathbb{Z})$, and let $E_{2}$ be the Eisenstein series of weight two for $\Gamma_{1}$. Then we obtain the map $\delta_{k}: M_{k}\left(\Gamma_{1}\right) \rightarrow M_{k+2}\left(\Gamma_{1}\right)$, known as the Serre derivative, by setting

$$
\delta_{k}(f)=\frac{d}{d z}-\frac{\pi i}{6} k E_{2} f
$$

for $f \in M_{k}\left(\Gamma_{1}\right)$. The goal of this paper is to introduce another type of operator on modular forms involving the derivative which carries modular forms to modular forms by using certain theta functions.

In [3] Dong and Mason used certain theta series $\theta_{k}$ for nonnegative integers $k$ associated to a vector $v \in \mathbb{C}^{2 \sigma}$ and a symmetric positive definite integral $2 \sigma \times 2 \sigma$ matrix $A$ with even diagonal entries in order to construct Jacobi-like forms studied by Cohen, Manin and Zagier (cf. [2], [6]). In this paper, given a nonnegative

Supported in part by a PDA award from the University of Northern Iowa

2010 Mathematics Subject Classification: primary: 11F11; secondary: 11F50 
integer $\alpha$, we prove that the operator

$$
\mathcal{L}_{\alpha}=\theta_{0}^{2} \frac{d}{d z}-\frac{2 \pi i \alpha}{v^{t} A v} \theta_{0} \theta_{2}
$$

determines the complex linear map

$$
\mathcal{L}_{\alpha}: M_{\alpha}\left(\Gamma_{0}(N)\right) \rightarrow M_{\alpha+2 \sigma+2}\left(\Gamma_{0}(N)\right)
$$

of modular forms for a congruence subgroup $\Gamma_{0}(N)$. The proof is carried out by using the fact that the coefficients of the Jacobi-like forms determined by $\theta_{k}$ are quasimodular forms.

\section{Jacobi-like forms and quasimodular forms}

Let $\mathcal{H}$ be the Poincaré upper half plane, and let $R$ be the ring of holomorphic functions on $\mathcal{H}$. The group $S L(2, \mathbb{R})$ acts on $\mathcal{H}$ as usual by linear fractional transformations, so that

$$
\gamma z=\frac{a z+b}{c z+d}
$$

for all $z \in \mathcal{H}$ and $\gamma=\left(\begin{array}{ll}a & b \\ c & d\end{array}\right) \in S L(2, \mathbb{R})$. By setting

$$
\mathfrak{J}(\gamma, z)=c z+d, \quad \mathfrak{K}(\gamma, z)=\frac{c}{c z+d},
$$

for the same $z$ and $\gamma$, we obtain the maps $\mathfrak{J}, \mathfrak{K}: S L(2, \mathbb{R}) \times \mathcal{H} \rightarrow \mathbb{C}$ which satisfy

$$
\mathfrak{J}\left(\gamma \gamma^{\prime}, z\right)=\mathfrak{J}\left(\gamma, \gamma^{\prime} z\right) \mathfrak{J}\left(\gamma^{\prime}, z\right), \quad \mathfrak{K}\left(\gamma, \gamma^{\prime} z\right)=\mathfrak{J}\left(\gamma^{\prime}, z\right)^{2}\left(\mathfrak{K}\left(\gamma \gamma^{\prime}, z\right)-\mathfrak{K}\left(\gamma^{\prime}, z\right)\right)
$$

for all $z \in \mathcal{H}$ and $\gamma, \gamma^{\prime} \in S L(2, \mathbb{R})$.

We denote by $R[[X]]$ the complex algebra of formal power series in $X$ with coefficients in $R$. If $\delta$ is a nonnegative integer, we set

$$
R[[X]]_{\delta}=X^{\delta} R[[X]],
$$

so that an element $\Phi(z, X) \in R[[X]]_{\delta}$ can be written in the form

$$
\Phi(z, X)=\sum_{k=0}^{\infty} \phi_{k}(z) X^{k+\delta}
$$

with $\phi_{k} \in R$ for each $k \geqslant 0$. Given a function $f \in R$, a formal power series $\Phi(z, X) \in R[[X]]$, an integer $\lambda$, and an element $\gamma \in S L(2, \mathbb{R})$, we set

$$
\begin{aligned}
\left(\left.f\right|_{\lambda} \gamma\right)(z) & =\mathfrak{J}(\gamma, z)^{-\lambda} f(z) \\
\left(\left.\Phi\right|_{\lambda} ^{J} \gamma\right)(z, X) & =\mathfrak{J}(\gamma, z)^{-\lambda} e^{-\mathfrak{K}(\gamma, z) X} \Phi\left(\gamma z, \mathfrak{J}(\gamma, z)^{-2} X\right),
\end{aligned}
$$

for $z \in \mathcal{H}$. 
We now consider a discrete subgroup $\Gamma$ of $S L(2, \mathbb{R})$. Then a modular form of weight $\lambda$ for $\Gamma$ is a holomorphic function $f \in R$ satisfying

$$
\left.f\right|_{\lambda} \gamma=f
$$

for all $\gamma \in \Gamma$. We denote by $M_{\lambda}(\Gamma)$ the space of such modular forms.

Definition 2.1. A formal power series $\Phi(z, X) \in R[[X]]$ is a Jacobi-like form of weight $\lambda$ for $\Gamma$ if it satisfies

$$
\left(\left.\Phi\right|_{\lambda} ^{J} \gamma\right)(z, X)=\Phi(z, X)
$$

for all $z \in \mathcal{H}$ and $\gamma \in \Gamma$.

We denote by $\mathcal{J}_{\lambda}(\Gamma)$ the space of Jacobi-like forms of weight $\lambda$ for $\Gamma$. Given a nonnegative integer $\delta$, let $\mathcal{J}_{\lambda}(\Gamma)_{\delta}$ be the subspace of $\mathcal{J}_{\lambda}(\Gamma)$ defined by

$$
\mathcal{J}_{\lambda}(\Gamma)_{\delta}=\mathcal{J}_{\lambda}(\Gamma) \cap R[[X]]_{\delta}
$$

where $R[[X]]_{\delta}$ is as in $(2.2)$.

Definition 2.2. Given integers $\lambda$ and $m$ with $m \geqslant 0$, an element $\psi \in R$ is a quasimodular form for $\Gamma$ of weight $\lambda$ and depth at most $m$ if there are functions $\psi_{0}, \psi_{1}, \ldots, \psi_{m} \in R$ satisfying

$$
\left(\left.\psi\right|_{\lambda} \gamma\right)(z)=\sum_{r=0}^{m} \psi_{r}(z) \mathfrak{K}(\gamma, z)^{r}
$$

for all $z \in \mathcal{H}$ and $\gamma \in \Gamma$, where $\mathfrak{K}(\gamma, z)$ is as in (2.1). We denote by $Q M_{\lambda}^{m}(\Gamma)$ the space of such quasimodular forms.

If $\psi \in Q M_{\lambda}^{m}(\Gamma)$ satisfies (2.4), for $0 \leqslant k \leqslant m$ we define the complex linear map

$$
\mathfrak{S}_{k}: Q M_{\lambda}^{m}(\Gamma) \rightarrow R
$$

by setting

$$
\mathfrak{S}_{k} \psi=\psi_{k}
$$

Then it induces the map

$$
\mathfrak{S}_{k}: Q P_{\lambda}^{m}(\Gamma) \rightarrow Q M_{\lambda-2 k}^{m-k}(\Gamma)
$$

(see e.g. [4]). In particular, $\mathfrak{S}_{m} \psi$ is a modular form belonging to $M_{\lambda-2 m}(\Gamma)=$ $Q M_{\lambda-2 m}^{0}(\Gamma)$.

Lemma 2.3. Given quasimodular forms

$$
\phi \in Q M_{\lambda}^{m}(\Gamma), \quad \psi \in Q M_{\mu}^{m}(\Gamma),
$$

the function

$$
\left(\mathfrak{S}_{m} \psi\right)\left(\mathfrak{S}_{m-1} \phi\right)-\left(\mathfrak{S}_{m} \phi\right)\left(\mathfrak{S}_{m-1} \psi\right)
$$

is a modular form belonging to $M_{\lambda+\mu-4 m+2}(\Gamma)$. 
Proof. Since $\mathfrak{S}_{m} \phi$ and $\mathfrak{S}_{m} \psi$ are modular forms with

$$
\mathfrak{S}_{m} \phi \in M_{\lambda-2 m}(\Gamma), \quad \mathfrak{S}_{m} \psi \in M_{\mu-2 m}(\Gamma),
$$

we see that

$$
\begin{aligned}
\left(\left(\left(\mathfrak{S}_{m} \psi\right) \phi-\right.\right. & \left.\left.\left(\mathfrak{S}_{m} \phi\right) \psi\right)\left.\right|_{\lambda+\mu-2 m} \gamma\right)(z) \\
& =\mathfrak{J}(\gamma, z)^{-\lambda-\mu+2 m}\left(\left(\mathfrak{S}_{m} \psi\right)(\gamma z) \phi(\gamma z)-\left(\mathfrak{S}_{m} \phi\right)(\gamma z) \psi(\gamma z)\right) \\
& =\left(\mathfrak{S}_{m} \psi\right)(z)\left(\left.\phi\right|_{\lambda} \gamma\right)(z)-\left(\mathfrak{S}_{m} \phi\right)(z)\left(\left.\psi\right|_{\mu} \gamma\right)(z) \\
& =\sum_{r=0}^{m-1}\left(\left(\mathfrak{S}_{m} \psi\right)(z)\left(\mathfrak{S}_{r} \phi\right)(z)-\left(\mathfrak{S}_{m} \phi\right)(z)\left(\mathfrak{S}_{r} \psi\right)(z)\right) \mathfrak{K}(\gamma, z)^{r}
\end{aligned}
$$

for all $\gamma \in \Gamma$ and $z \in \mathcal{H}$. In particular,

$$
\left(\mathfrak{S}_{m} \psi\right) \phi-\left(\mathfrak{S}_{m} \phi\right) \psi
$$

is a quasimodular form belonging to $Q M_{\lambda+\mu-2 m}^{m-1}(\Gamma)$; hence it follows that

$$
\mathfrak{S}_{m-1}\left(\left(\mathfrak{S}_{m} \psi\right) \phi-\left(\mathfrak{S}_{m} \phi\right) \psi\right)=\left(\mathfrak{S}_{m} \psi\right)\left(\mathfrak{S}_{m-1} \phi\right)-\left(\mathfrak{S}_{m} \phi\right)\left(\mathfrak{S}_{m-1} \psi\right)
$$

is an element of $M_{\lambda+\mu-4 m+2}(\Gamma)$.

Quasimodular forms are linked to Jacobi-like forms due to the fact that each coefficient of a Jacobi-like form is a quasimodular form. To be more precise, we consider a Jacobi-like form $\Phi(z, X) \in \mathcal{J}_{\lambda}(\Gamma)_{\delta}$ as in $(2.3)$, and set

$$
\Pi_{m}^{\delta} \Phi(z, X)=\phi_{m}
$$

for a nonnegative integer $m$. Then this formula determines a complex linear map

$$
\Pi_{m}^{\delta}: \mathcal{J}_{\lambda}(\Gamma)_{\delta} \rightarrow Q M_{\lambda+2 m+2 \delta}^{m}(\Gamma)
$$

such that

$$
\left(\mathfrak{S}_{k} \circ \Pi_{m}^{\delta}\right) \Phi(z, X)=\mathfrak{S}_{k}\left(\phi_{m}\right)=\frac{1}{k !} \phi_{m-k}
$$

for $0 \leqslant k \leqslant m$ with $\mathfrak{S}_{k}$ being as in (2.5). Furthermore, there is a right inverse to this surjective map, which can be regarded as a lifting map from quasimodular forms to Jacobi-like forms (cf. [1]).

\section{Theta functions}

We fix a positive integer $\sigma$, an element $v$ of $\mathbb{C}^{2 \sigma}$ regarded as a column vector, and a symmetric positive definite integral $2 \sigma \times 2 \sigma$ matrix $A$ whose diagonal entries are even. For each nonnegative integer $k$, we consider the theta function $\theta_{k}: \mathcal{H} \rightarrow \mathbb{C}$ by

$$
\theta_{k}(z)=\sum_{\ell \in \mathbb{Z}^{2 \sigma}}\left(v^{t} A \ell\right)^{k} e^{\pi i\left(\ell^{t} A \ell\right) z}
$$


for all $z \in \mathcal{H}$ studied by Dong and Mason in [3]. We then define the formal power series $\Theta(z, X) \in R[[X]]$ associated to the sequence $\left\{\theta_{n}\right\}_{n=0}^{\infty}$ of such theta functions by

$$
\Theta(z, X)=\sum_{n=0}^{\infty} \frac{2^{n}(2 \pi i)^{n}}{(2 n) !} \theta_{2 n}(z) X^{n}
$$

for all $z \in \mathcal{H}$.

Let $N$ be the smallest positive integer such that $N A^{-1}$ is an integral matrix with even diagonal entries, and let $\Gamma_{0}(N) \subset S L(2, \mathbb{Z})$ be the associated congruence subgroup given by

$$
\Gamma_{0}(N)=\left\{\left(\begin{array}{ll}
a & b \\
c & d
\end{array}\right) \in S L(2, \mathbb{Z}) \mid c \equiv 0 \quad(\bmod N)\right\} .
$$

We consider the character $\varepsilon$ on the set of positive integers defined by the Jacobi symbol

$$
\varepsilon(n)=\left(\frac{(-1)^{\sigma} \operatorname{det} A}{n}\right)
$$

for each positive integer $n$.

Theorem 3.1 (Dong-Mason). The formal power series $\Theta(z, X) \in R[[X]]$ given by (3.2) satisfies the transformation property

$$
\Theta\left(\gamma z, \mathfrak{J}(\gamma, z)^{-2} X\right)=\varepsilon(d) \mathfrak{J}(\gamma, z)^{\sigma} \exp \left[v^{t} A v \mathfrak{K}(\gamma, z) X\right] \cdot \Theta(z, X)
$$

for all $z \in \mathcal{H}$ and $\gamma \in \Gamma_{0}(N)$, where $\mathfrak{J}(\gamma, z)$ and $\mathfrak{K}(\gamma, z)$ are as in (2.1) and $d$ is the $(2,2)$-entry of the matrix $\gamma$.

Proof. See [3].

We now consider the theta function given by (3.1) for $k=0,2$ associated to the vector $v \in \mathbb{C}^{2 \sigma}$ and the symmetric integral matrix $A$ with $v^{t} A v \neq 0$, and set

$$
\mathcal{L}_{\alpha}=\theta_{0}^{2} \frac{d}{d z}-\frac{2 \pi i \alpha}{v^{t} A v} \theta_{0} \theta_{2}
$$

for a nonnegative integer $\alpha$.

Theorem 3.2. The differential operator given by (3.5) determines the complex linear map

$$
\mathcal{L}_{\alpha}: M_{\alpha}\left(\Gamma_{0}(N)\right) \rightarrow M_{\alpha+2 \sigma+2}\left(\Gamma_{0}(N)\right)
$$

for each $\alpha \geqslant 0$.

Proof. By taking the square of $\Theta(z, X)$ and using (3.4), it can be shown easily that the formal power series $\vartheta(z, X) \in R[[X]]$ given by

$$
\vartheta(z, X)=\sum_{n=0}^{\infty} \sum_{\ell=0}^{n}\left(\frac{2 \pi i}{v^{t} A v}\right)^{n} \frac{\theta_{2 \ell}(z) \theta_{2 n-2 \ell}(z)}{(2 \ell) !(2 n-2 \ell) !} X^{n}
$$


is a Jacobi-like form belonging to $\mathcal{J}_{2 \sigma}\left(\Gamma_{0}(N)\right)_{0}$. Thus, if we set

$$
\xi_{n}=\sum_{\ell=0}^{n}\left(\frac{2 \pi i}{v^{t} A v}\right)^{n} \frac{\theta_{2 \ell} \theta_{2 n-2 \ell}}{(2 \ell) !(2 n-2 \ell) !}
$$

for $n \geqslant 0$ and use the linear map (2.6) for $\lambda=2 \sigma$ and $\delta=0$, we see that

$$
\Pi_{m}^{0} \vartheta(z, X)=\xi_{m} \in Q M_{2 \sigma+2 m}^{m}\left(\Gamma_{0}(N)\right)
$$

with

$$
\mathfrak{S}_{r}\left(\xi_{m}\right)=\frac{1}{r !} \xi_{m-r}=\frac{1}{r !}\left(\frac{2 \pi i}{v^{t} A v}\right)^{m-r} \sum_{\ell=0}^{m-r} \frac{\theta_{2 \ell} \theta_{2 m-2 r-2 \ell}}{(2 \ell) !(2 m-2 r-2 \ell) !}
$$

for $0 \leqslant r \leqslant m$. In particular, we have

$$
\begin{aligned}
\mathfrak{S}_{m}\left(\xi_{m}\right) & =\frac{1}{m !} \theta_{0}^{2} \\
\mathfrak{S}_{m-1}\left(\xi_{m}\right) & =\frac{1}{(m-1) !}\left(\frac{2 \pi i}{v^{t} A v}\right) \sum_{\ell=0}^{1} \frac{\theta_{2 \ell} \theta_{2-2 \ell}}{(2 \ell) !(2-2 \ell) !}=\frac{1}{(m-1) !}\left(\frac{2 \pi i}{v^{t} A v}\right) \theta_{0} \theta_{2} .
\end{aligned}
$$

We now consider a modular form

$$
f \in M_{\alpha}\left(\Gamma_{0}(N)\right)
$$

satisfying

$$
f(\gamma z)=\mathfrak{J}(\gamma, z)^{\alpha} f(z)
$$

for all $\gamma \in \Gamma_{0}(N)$ and $z \in \mathcal{H}$. By taking its derivative with respect to $z$ and using the identity

$$
\frac{d}{d z} \mathfrak{J}(\gamma, z)=\mathfrak{J}(\gamma, z) \mathfrak{K}(\gamma, z)
$$

we obtain

$$
\mathfrak{J}(\gamma, z)^{-2} f^{\prime}(\gamma z)=\alpha \mathfrak{J}(\gamma, z)^{\alpha} \mathfrak{K}(\gamma, z) f(z)+\mathfrak{J}(\gamma, z)^{\alpha} f^{\prime}(z)
$$

Thus, if we set

$$
\eta=\frac{f^{\prime}}{f},
$$

we have

$$
\begin{aligned}
\eta(\gamma z) & =\frac{\mathfrak{J}(\gamma, z)^{2}\left(\alpha \mathfrak{J}(\gamma, z)^{\alpha} \mathfrak{K}(\gamma, z) f(z)+\mathfrak{J}(\gamma, z)^{\alpha} f^{\prime}(z)\right)}{\mathfrak{J}(\gamma, z)^{\alpha} f(z)} \\
& =\mathfrak{J}(\gamma, z)^{2}\left(\frac{f^{\prime}(z)}{f(z)}+\alpha \mathfrak{K}(\gamma, z)\right) \\
& =\mathfrak{J}(\gamma, z)^{2}(\eta(z)+\alpha \mathfrak{K}(\gamma, z)) .
\end{aligned}
$$


for all $\gamma \in \Gamma_{0}(N)$ and $z \in \mathcal{H}$; hence we see that

$$
\left(\left.\eta\right|_{2} \gamma\right)(z)=\eta(z)+\alpha \mathfrak{K}(\gamma, z)
$$

Thus the $m$-th power $\eta^{m}$ of $\eta$ for a positive integer $m$ satisfies

$$
\left(\left.\eta^{m}\right|_{2 m} \gamma\right)(z)=\sum_{r=0}^{m} \alpha^{r}\left(\begin{array}{c}
m \\
r
\end{array}\right)\left(\frac{f^{\prime}(z)}{f(z)}\right)^{m-r} \mathfrak{K}(\gamma, z)^{r}
$$

for all $\gamma \in \Gamma_{0}(N)$ and $z \in \mathcal{H}$. In particular, $\eta^{m}$ is a quasimodular form belonging to $Q M_{2 m}^{m}\left(\Gamma_{0}(N)\right)$ with

$$
\mathfrak{S}_{m}\left(\eta^{m}\right)=\alpha^{m}, \quad \mathfrak{S}_{m-1}\left(\eta^{m}\right)=m \alpha^{m-1} f^{\prime} / f .
$$

From this, (3.7) and (3.8) we obtain

$$
\begin{aligned}
\mathfrak{S}_{m}\left(\xi_{m}\right) \mathfrak{S}_{m-1}\left(\eta^{m}\right)-\mathfrak{S}_{m}\left(\eta^{m}\right) \mathfrak{S}_{m-1}\left(\xi_{m}\right) & \\
= & \frac{1}{m !}\left(\theta_{0}^{2} m \alpha^{m-1} \frac{f^{\prime}}{f}-m\left(\frac{2 \pi i}{v^{t} A v}\right) \theta_{0} \theta_{2} \alpha^{m}\right) \\
& =\frac{\alpha^{m-1}}{(m-1) ! f}\left(\theta_{0}^{2} f^{\prime}-\frac{2 \pi i \alpha}{v^{t} A v} \theta_{0} \theta_{2} f\right),
\end{aligned}
$$

which belongs to $M_{\alpha+2 \sigma+2}\left(\Gamma_{0}(N)\right)$ by Lemma 2.3 ; hence it follows that $\mathcal{L}_{\alpha} f \in$ $M_{\alpha+2 \sigma+2}\left(\Gamma_{0}(N)\right)$.

Remark 3.3. If $\varepsilon$ is the character given by (3.3) and if $\chi$ is the corresponding character on $\Gamma_{0}(N)$, the theta series $\theta_{0}$ is a modular form for $\Gamma_{0}(N)$ of weight $\sigma$ with character $\chi$ (cf. [5]). Using this and Theorem 3.2, we see that the operator

$$
\widehat{\mathcal{L}}_{\alpha}=\theta_{0} \frac{d}{d z}-\frac{2 \pi i \alpha}{v^{t} A v} \theta_{2}
$$

determines the linear map

$$
\widehat{\mathcal{L}}_{\alpha}: M_{\alpha}\left(\Gamma_{0}(N)\right) \rightarrow M_{\alpha+\sigma+2}\left(\Gamma_{0}(N), \chi\right),
$$

where $M_{\alpha+\sigma+2}\left(\Gamma_{0}(N), \chi\right)$ denotes the space of modular forms for $\Gamma_{0}(N)$ of weight $\alpha+\sigma+2$ with character $\chi$.

\section{References}

[1] Y. Choie and M.H. Lee, Quasimodular forms, Jacobi-like forms, and pseudodifferential operators, preprint.

[2] P.B. Cohen, Y. Manin, and D. Zagier, Automorphic pseudodifferential operators, Algebraic Aspects of Nonlinear Systems, Birkhäuser, Boston, 1997, pp. 17-47.

[3] C. Dong and G. Mason, Transformation laws for theta functions, CRM Proc. Lecture Notes, vol. 30, Amer. Math. Soc., Providence, RI, 2001, pp. 15-26. 
[4] F. Martin and E. Royer, Formes modulaires et transcendance, Formes modulaires et périodes (S. Fischler, E. Gaudron, and S. Khémira, eds.), Soc. Math. de France, 2005, pp. 1-117.

[5] B. Schoeneberg, Elliptic modular functions, Springer-Verlag, Heidelberg, 1974.

[6] D. Zagier, Modular forms and differential operators, Proc. Indian Acad. Sci. Math. Sci. 104 (1994), 57-75.

Address: Min Ho Lee: Department of Mathematics, University of Northern Iowa, Cedar Falls, IA 50614, U.S.A.

E-mail: lee@math.uni.edu

Received: 21 August 2013 